\title{
Evaluación neuropsicológica de las dificultades de aprendizaje lectoescritoras en
}

\author{
el aula
}

\section{Neuropsychological evaluation of learning disabilities in the classroom}

\author{
Cláudia Balado, Rosa Maria Rivas, Santiago Torres López,. Eva María Taboada \\ Universidad de Santiago de Compostela
}

\begin{abstract}
Resumen
Las dificultades lectoescritoras -dislexia- afectan a un $5-10 \%$ de los niños en edad escolar. Se trata de un trastorno que conlleva múltiples consecuencias negativas en los diferentes ámbitos del desarrollo. Para conseguir reducir dicha prevalencia y mejorar los resultados de las intervenciones, se hace necesaria la detección precoz. Para ello, se necesita de una evaluación temprana capaz de detectar a aquellos niños en riesgo de desarrollar el trastorno. De acuerdo con ello, el objetivo de este trabajo ha sido confeccionar, desde la perspectiva de la neuropsicología escolar, una propuesta de evaluación para la detección temprana de estas dificultades.

Palabras clave: dificultades de aprendizaje, lectoescritura, dislexia, evaluación, neuropsicología escolar.
\end{abstract}

\begin{abstract}
Dyslexia disorder affects 5-10\% of children of school age. It is a disorder that involves multiple negative consequences. Early detection is necessary to achieve reduce prevalence and improve outcomes of interventions. To do this, it is need an early evaluation that can detect those children at risk of developing the disorder. Accordingly, the objective of this work has been make an evaluation proposal for early detection of literacy difficulties, from the perspective of school neuropsychology.

Keywords: Learning difficulties, Literacy, Dyslexia, Evaluation, School neuropsychology.
\end{abstract}

\section{Introducción}

En la actualidad, se estima una prevalencia de un $5-15 \%$ de niños en edad escolar que presentan trastorno específico del aprendizaje (APA, 2014) y, entre un 5-10\% con dislexia (Málaga y cols., 2010, citado por Mateos y López, 2011; Preilowsky y Matute, 2011).

En la actualidad, el trastorno específico de aprendizaje genera en los sujetos que lo manifiestan múltiples consecuencias negativas: baja autoestima (Camargo, 2010; Mateos y López, 2011); inseguridad, frustración, rechazo a las tareas del ámbito académico (Camargo, 2010); una imagen de sí mismos más negativa a nivel general, menor motivación para el aprendizaje escolar (González-Pineda y cols., 2000, citado por Mateos y López, 2011); apatía, problemas de comportamiento (Mateos y López, 2011); retraimiento social (Preilowski y Matute, 2011); ... "logros académicos bajos, tasas más altas de abandono de la educación secundaria, bajos porcentajes de educación secundaria, altos niveles de malestar psicológico, problemas de salud mental general, tasas más altas de desempleo o infraempleo e ingresos más bajos" (APA, 2014, p. 73).

Las dificultades lectoescritoras no afectan sólo al proceso de enseñanza-aprendizaje, sino al desarrollo global y a la adaptación del individuo (Camargo, 2010). En contrapartida, el éxito en la lectura tiene una influencia positiva en otras áreas académicas pues contribuye a aumentar el desarrollo social y, en general, el placer por la escuela (Stanovich, 1994, citado por Pogorzelski y Wheldall, 2005).

La importancia del estudio de este trastorno estriba en la posibilidad de realizar una adecuada evaluación e intervención tempranas, dado que la tasa de éxito es mayor cuanto menor sea el sujeto. De igual modo, cuanto antes se inicie la intervención, más significativos serán los avances (Preilowski y Matute, 2011). Por el contrario, cuando el tratamiento se inicia después de $4^{\circ}$ de primaria, el pronóstico es menos optimista (Preilowski y Matute, 2011).

En definitiva, no puede obviarse la importancia de un tema tan significativo y actual como son las dificultades de aprendizaje. Por ello, en este trabajo, de acuerdo con el estudio previo de las características de la dislexia, investigadas las variables predictoras identificadas en la actualidad, así como los diversos modelos de evaluación, se desarrolla el objetivo principal de este trabajo: planificar y diseñar, en el marco de la perspectiva de la neuropsicología escolar, una propuesta de evaluación de las dificultades de aprendizaje en la lectoescritura, dirigida a la detección temprana de niños en riesgo de presentar dichas dificultades en el futuro.

\section{Desarrollo teórico}

Factores causales de las dificultades lectoescritoras
La dislexia del desarrollo es un trastorno complejo
cuya génesis es multifactorial. En la actualidad, se
desconocen las causas, si bien están establecidas aquellas
condiciones que no lo son: recordemos que para
diagnosticar a un individuo con dificultades de
aprendizaje éste debe presentar una inteligencia normal y
una educación y ambiente sociocultural adecuados. Los


estudios de los últimos años han tratado de arrojar luz sobre el tema, sin embargo, por el momento se conocen correlaciones pero no causas.

El aprendizaje y el desarrollo son fruto de un proceso complejo en el cual intervienen factores tanto biológicos como psicológicos y sociales. En concordancia, las dificultades de aprendizaje son también resultado de un proceso multifactorial, que ha de considerarse, por tanto, desde una perspectiva multidisciplinar.

Tras las dificultades de aprendizaje existe una alteración en los procesos psicológicos básicos, evolutivamente ligados a una disfunción del sistema nervioso central (SNC). Dado que el problema es una disfunción en el SNC, resulta trascendente su evaluación neuropsicológica.

En los últimos años, la relevancia de la neuropsicología escolar ha ido en aumento, en parte gracias al desarrollo de las técnicas de neuroimagen, que han permitido conocer más sobre el desarrollo anatómico y funcional del cerebro, así como sus relaciones con la progresiva adquisición y organización de habilidades cognitivas (Carboni-Román y cols., 2006). Si bien, estos conocimientos no implican que las dificultades de aprendizaje sean consecuencia única de una causa orgánica. Lo que sí se sabe es que se relacionan variables tanto biológicas como ambientales con el desarrollo de estas dificultades.

\section{Factores intrínsecos o neuropsicológicos}

Para poder llevar a cabo todo el proceso lectoescritor, el niño debe desarrollar primero ciertas funciones básicas visuales, auditivas y sensorio-motoras (Preilowski y Matute, 2011), así como cognitivas. A continuación, se comentan los principales factores intrínsecos estudiados en relación a las dificultades en el aprendizaje de la lectura y la escritura.

Factores psicomotores. Ciertos estudios muestran que es común encontrar déficits motores y ejecutivos en niños que presentan dislexia (Henry, Messer y Nash, 2012, citado por Thompson y cols., 2015).

Factores cognitivos. La base para todo aprendizaje es la percepción y esto no difiere en la adquisición de la habilidad lectora y escritora, que requiere de una madurez perceptiva fundamentalmente en las áreas visual y auditiva (Bravo, 2004). Para el aprendizaje de la lectura y la escritura será indispensable que el individuo presente las siguientes destrezas: discriminación auditiva y visual, integración auditiva, memoria auditiva y visual, figura-fondo visual, constancia de la forma,...

Factores de personalidad o socioafectivos $\mathrm{Al}$ igual que el resto de componentes, no pueden considerarse aisladamente, sino que siempre se encuentran en interacción con el resto de variables. En ocasiones, no puede determinarse si los factores son causales o simples correlaciones. Miranda, Vidal-Abarca y Soriano (2000) se han centrado en el análisis de algunos de los factores socioafectivos relacionados con las dificultades de aprendizaje. Exponen que diversos estudios indican que los individuos con estas dificultades presentan un estilo atribucional desadaptativo, un bajo autoconcepto, niveles mayores de ansiedad y depresión que aquellos sin dificultades de aprendizaje, una conducta más hiperactiva y mayores déficits en la autorregulación. En el DSM-5, se habla también de una mayor conducta negativista en estos niños (APA, 2014).

Factores lectoescritores A pesar de que numerosos estudios se centran en áreas implicadas en este proceso, la mayoría de autores coinciden en que los problemas de lectura en la dislexia no se asocian con la disfunción de un área concreta, sino con un patrón de funcionalidad atípico (Carboni- Román y cols., 2006). Esto es, se defiende que la anomalía no se encuentra en una región particular, sino que es debido más bien a una conexión deficiente entre las áreas críticas (Preilowski y Matute, 2011).

Los hallazgos recientes muestran que los sujetos disléxicos tienen problemas perceptivos auditivos, fonológicos o mezcla de ambos (Galaburda y Cestnick, 2003). Ello provoca dificultades en las correspondencias entre fonemas y grafemas.

Dado que uno de los factores que predomina son las dificultades fonológicas, muchos estudios se centran en esta cuestión. Un aspecto esencial es la conciencia fonológica. Goswani (2009) afirma que la conciencia fonológica es un predictor de la adquisición de la lectura en todas las lenguas estudiadas. Los individuos que no presenten un buen procesamiento fonológico, no automatizarán con tanta rapidez las reglas de conversión grafema-fonema, tan importantes para la decodificación (Suarez-Coalla, Garcia-de-Castro, y Cuetos, 2013).

Además de la conversión grafema-fonema, en la lectura es importante el acceso al léxico, pues tal y como señalan Suárez-Coalla et al. (2013), para conseguir leer de manera fluida es necesario presentar un adecuado nivel de velocidad en el acceso al léxico. En sujetos con dislexia, se ha comprobado que la velocidad de nombrar aparece deficitaria (Escribano, 2007, citado por Aguilar Villagrán, 2010).

Gallagher, Frith y Snowling (2000) examinaron distintos posibles precursores de dislexia, encontrando que el predictor más fuerte de la lectoescritura a los 6 años era el conocimiento de las letras a los 45 meses.

Estas tres variables (conocimiento de letras, velocidad de nombrado y conciencia fonológica) han sido ampliamente estudiadas y se sabe que predicen la capacidad de los niños para decodificar y reconocer palabras y leer fluidamente (Caravolas y cols., 2012, citado por Thompson y cols., 2015).

\section{Factores extrínsecos}

Las dificultades en la lectura y la escritura no pueden ser consideradas simplemente como consecuencia de factores intrínsecos, pues ésta sería una visión reduccionista; sino que, como ya se ha mencionado, poseen una etiología multifactorial en la que intervienen variables tanto biológicas como ambientales.

Factores de la familia Uno de los factores de riesgo que se ha estudiado en las dificultades de aprendizaje (tanto en lectoescritura como en matemáticas) es el hecho de ser prematuro (Johnson y cols., 2011), o nacer con muy bajo peso (APA, 2014). También se ha investigado cómo aumenta el riesgo de padecer un trastorno específico del aprendizaje el hecho de estar expuesto a la nicotina en la etapa prenatal (APA, 2014). 
El $72 \%$ de los niños que presentan dislexia tienen un familiar de primer grado con la misma dificultad. Asimismo se conoce que un niño cuyo padre es disléxico presenta una probabilidad 8 veces mayor de padecer dislexia, en relación a la población media (Camargo, 2010). No obstante, debemos considerar que, además del vínculo genético, influye también la conducta de la familia. Así, las prácticas educativas que desarrolla la familia son importantes, pues ello repercutirá en una mayor o menor disposición del alumno hacia el aprendizaje, la lectura y la escritura y facilitará o no el proceso (Preilowski y Matute, 2011; Suárez-Coalla et al., 2013).

Factores del aula Es de vital importancia tener en consideración la metodología que se utiliza para el proceso de enseñanza-aprendizaje de la lectura y la escritura. Para llegar a obtener el significado de un texto leído deben darse diversas operaciones: reconocimiento de la información, relación de esa información con la previamente almacenada por el sujeto, activación de los significados semánticos, relacionar las distintas partes del texto, construcción del significado global o macroestructura, entre otras (De Ramírez, 2000).

Por ello, es importante el uso de un método mixto, que a la vez tenga en cuenta los puntos fuertes de cada alumno, para a partir de éstos, fortalecer los débiles. Algunos autores, sugieren que un buen método de enseñanza debe exponer al niño a claves informativas simultáneas, de modo que puedan establecer un léxico ortográfico consistente y un conjunto de reglas de conversión grafema- fonema a partir de las regularidades ortográficas de la lengua (citado por López-Higes y cols., 2003). Es decir, debe promocionarse el uso de la ruta fonológica y la ruta visual por igual. Para ello será necesario el uso de vocabulario familiar para el niño, en un contexto motivante (López-Higes y cols., 2003).

Además, la escuela debe facilitar un buen ambiente lector (Suárez, Manso y Godoy 2010). Es importante fomentar la motivación y la actitud positiva hacia el aprendizaje de la lectura y la escritura, por ejemplo, propiciando momentos de lectura en el aula en los cuales los maestros leen en un primer momento, sirviendo así como modelos a los alumnos. Suárez et al. (2010) proponen también acondicionar un "rincón de lectura" en la clase así como disponer de material variado y actividades complementarias de animación lectora.

Evaluación de las dificultades lectoescritoras

El diagnostico de las dificultades de aprendizaje no es un proceso sencillo, entre otras razones porque ya, durante la etapa infantil, hay evoluciones que son muy particulares en los niños y cada uno presenta variaciones individuales en su proceso madurativo.

Como señalan Thompson y cols. (2015), en la evaluación deben tenerse en cuenta diferentes factores simultáneamente. De lo contrario, los modelos que sólo consideran unos pocos, reducen la sensibilidad y/o especificidad de la evaluación, conllevando a un sobre o infradiagnóstico.

No hay indicadores universales que puedan sustentar claramente el diagnóstico. Por eso, en ciertas ocasiones, puede resultar una tarea confusa. Una evaluación comprehensiva debe incluir métodos de medida diversos, tanto formales como informales, así como analizar diferentes contextos y escenarios. Ha de llevarse a cabo una evaluación del desarrollo cognitivo, emocional y social. Igualmente, como defienden D'Amato y cols. (2005), debe centrarse en las necesidades del niño.

Desde la neuropsicología escolar, se defiende la evaluación, diagnóstico e intervención de sujetos que tienen dificultades en sus aprendizajes $\mathrm{y}$, como consecuencia, muestran trastornos de comportamiento en su centro escolar. Por ello, en los últimos años se viene recomendando una evaluación neuropsicológica comprehensiva de cara a la intervención en el centro escolar. Esta perspectiva tiene en cuenta que el ambiente influye en la génesis de las dificultades y que es igualmente importante en la búsqueda de soluciones (Restrepo, 2015). Esto es, además de la evaluación de capacidades neuropsicológicas y cognitivas, es necesaria la exploración de la familia y del ambiente educativo, principalmente (Witsken y cols., 2008, citado por Manga y Ramos, 2011).

La evaluación estandarizada permite consenso entre los profesionales. Su función es diagnóstica; esto es, busca la confirmación de un diagnóstico de trastorno específico del aprendizaje a través de la evaluación de diversas medidas utilizadas universalmente por diferentes expertos. Evalúan determinados aspectos que se sabe que influyen, pero como ya se ha visto, existen factores muy variados según los individuos y posiblemente diferentes subtipos, por lo que una prueba estandarizada es improbable que alcance a analizar todo ello, si bien no podemos prescindir de éstas.

La finalidad de una evaluación cualitativa es la detección precoz de aquellos sujetos que se encuentran en una situación de riesgo de padecer dificultades de aprendizaje, para, de ese modo, intervenir preventivamente antes de que desarrollen dichas dificultades, consiguiéndose así una optimización del desarrollo de esos niños.

La evaluación no debe centrarse simplemente en los errores, sino que ha de posibilitar la realización de un perfil tanto de las debilidades como las fortalezas cognitivas y comportamentales de cada niño (Preilowski y Matute, 2011). Asimismo, debe estar centrada en el proceso y no tan sólo en el resultado (Báez Ávila y cols., 2009). El objetivo de una buena evaluación es conseguir realizar un plan de intervención individualizado.

Es importante destacar la necesidad de una evaluación y una intervención multidisciplinares, interdependientes y relacionadas directamente. Los modelos basados en la respuesta a la intervención (Jiménez y cols., 2011) defienden esta postura. Estos modelos han surgido como crítica al modelo del déficit, y se centran en evaluar la respuesta del alumno a una instrucción adecuada.

Teniendo en cuenta ciertas limitaciones de estos modelos, autores como Kavale y cols. (2005) proponen un modelo jerárquico de evaluación.

\section{Desarrollo metodológico}

\section{Procedimiento}

Para la elaboración de la propuesta de evaluación de las dificultades lectoescritoras, en un primer momento, se 
ha llevado a cabo una revisión teórica actualizada sobre los principales factores de riesgo tras las dificultades lectoescritoras y los modelos de evaluación de las mismas desde la orientación neuropsicológica. Para ello, se ha procedido a una búsqueda bibliográfica sistemática utilizando las siguientes bases de datos: Dialnet, PsycINFO, WOS (Web of Science) y SCOPUS; y los siguientes metabuscadores: Google y Google Académico. En un segundo momento, se ha procedido a una revisión de los tests más usados en el campo de la evaluación de los diferentes aspectos de las dificultades lectoescritoras. Para efectuar dicha revisión, se ha hecho una búsqueda en las principales editoriales; a saber, TEA, CEPE y EOS.

Una vez comentados y explicados los factores de riesgo conocidos hasta la actualidad y revisados los principales test utilizados, se ha desarrollado la propuesta de evaluación que aparece a continuación.

Destinatarios. En nuestro país, el sistema educativo regla el aprendizaje de la lectura y la escritura en el primer curso de educación primaria. Puesto que con la siguiente propuesta de evaluación se pretende una detección temprana, se destinará a sujetos de menor edad, antes de que comiencen dicho aprendizaje. Por ello, la evaluación se propone para niños de entre 3 y 6-8 años. Se extiende hasta los 8 años dado que no todos los niños realizan el aprendizaje de la lectoescritura al mismo tiempo. Asimismo, es posible que algún niño no manifieste riesgo hasta que realmente comience ese proceso de aprendizaje.

Instrumento. El protocolo de evaluación (Tabla 1) presta atención a los factores causales explicados anteriormente así como a los criterios explicitados por el DSM-5. Como ya se ha mencionado, sigue el esquema propuesto por Kavale y Forness en el 2000 (citado por Kavale y cols., 2005), dentro de la perspectiva de la neuropsicología escolar. La discrepancia entre habilidad y rendimiento será identificada en un primer momento por el profesor/a. Puesto que el sujeto todavía no ha comenzado el aprendizaje de la lectura ni la escritura, no podrán evaluarse los déficits en dichas áreas, pero sí en los procesos subyacentes, así como la eficiencia del aprendizaje. A continuación, ha de asegurarse que las dificultades que presente el individuo no se deban a ningún otro diagnóstico como daño sensorial, trastorno emocional, instrucción inadecuada o diferencias culturales.

Para llevar a cabo la evaluación se hablará tanto con los padres como con los niños y los profesores. Se trata de una evaluación cualitativa, por ello, la observación y las entrevistas serán métodos esenciales. No obstante, la evaluación cuantitativa es imprescindible y es por eso que se usarán ambas.

La propuesta de evaluación consta de 6 partes. Las dos primeras recogen variables de identificación, tanto del sujeto como de la familia. La tercera indaga sobre el historial evolutivo del individuo, puesto que se sabe que anomalías durante el embarazo o el parto, presencia de familiares con el diagnóstico o retrasos evolutivos en diferentes áreas, pueden afectar a las dificultades de aprendizaje. Seguidamente, las tres partes restantes evalúan los factores de riesgo de las dificultades de aprendizaje de la lectura y la escritura. Así pues, se analizan los factores intrínsecos, neuropsicológicos o del sujeto, así como los extrínsecos, incluyendo estos la familia y el aula.

Tabla 1.

Propuesta de evaluación de las dificultades lectoescritoras

\begin{tabular}{|c|c|c|}
\hline \multicolumn{3}{|c|}{ 1. Variables de identificación del sujeto: } \\
\hline $\begin{array}{l}\text { Nombre y apellidos: } \\
\text { de nacimiento: }\end{array}$ & Sexo: & Edad/ Fecha \\
\hline $\begin{array}{l}\text { Curso actual: } \\
\text { acude: }\end{array}$ & & Colegio al que \\
\hline
\end{tabular}
acude:

2. Historial evolutivo

Problemas durante el embarazo y parto

Ha sufrido intervenciones quirúrgicas:

Ha sufrido accidentes graves:

Sufre otitis de

repetición:

Ha estado ingresado en alguna ocasión (motivos):

Algún familiar presenta problemas médicos:

Edad a la que comenzó a andar: Edad a la que comenzó a hablar:

Edad a la que controló los esfínteres: Edad a la que fue escolarizado:

Acudió a guardería:

Sociabilidad. Juegos y hobbies (ej. le gusta que le lean cuentos):

Ha sido visto por otros especialistas:

\section{Prerrequisitos/ Factores de riesgo del sujeto:}

a) Evaluación de la psicomotricidad (se usará la observación, la entrevista con el niño, familia y profesor y medidas objetivas:

Evaluación de la motricidad (se incluye motricidad fina y gruesa, equilibrio, coordinación, fuerza y coordinación viso-motriz):

Evaluación del esquema corporal:

Evaluación de la lateralidad:

Evaluación de la estructuración espacio-temporal

b) Evaluación de aspectos cognitivos (se usará la observación, la entrevista con el niño, familia y profesor y medidas objetivas:

Evaluación de la percepción visual y auditiva (se incluye recepción visual, asociación visual, discriminación visual, figura-fondo visual, cierre visual, memoria visual, constancia perceptual visual, seriación, integración, recepción auditiva, asociación auditiva, discriminación auditiva, figura-fondo auditiva, cierre y fusión auditiva, memoria auditiva):

Evaluación de la atención:

Evaluación de la memoria (principalmente memoria sensorial y memoria de trabajo):

c) Evaluación de la personalidad (se usará la observación, la entrevista con el niño, familia y profesor y medidas objetivas -consultar anexos para seleccionar test para dicha evaluación-):

Evaluación de aspectos emocionales (principalmente síntomas ansiosos y/o depresivos)

Evaluación de habilidades sociales:

Evaluación de la autorregulación:

Evaluación de la tolerancia a la frustración:

Evaluación del estilo atribucional:

Evaluación de la autoestima y el autoconcepto:

Evaluación de la conducta (principalmente, evaluar si es hiperactiva y/o negativista)

d) Evaluación de aspectos relacionados con la lectoescritura (se usará la observación, la entrevista con el niño, familia y profesor y medidas objetivas -consultar anexos para seleccionar test para dicha 
evaluación-):

Evaluación del conocimiento de letras:

Evaluación de la velocidad de nombrar:

Evaluación de la conciencia fonológica:

Evaluación del nivel de vocabulario:

4. Evaluación de la familia y el ambiente familiar (se usará la entrevista con la familia y el niño así como medidas objetivas -consultar anexos para seleccionar test para dicha evaluación-):

Evaluación de la actitud hacia la lectura en la familia:

Evaluación de la disponibilidad de oportunidades para la lectura (libros en casa, tiempos de lectura, etc.)

Evaluación de prácticas educativas de la familia (juegos que estimulen el desarrollo fonológico, lectura de cuentos en casa, promoción de la autonomía):

Observaciones:

5. Evaluación de la metodología usada en el aula (se usará la entrevista con el profesor y el niño así como medidas objetivas -consultar anexos para seleccionar test para dicha evaluación-):

Metodología analítica, sintética o mixta:

Ambiente en el aula (de estrés, de diversión, estimulante, motivador):

Tiempos y espacios dedicados al aprendizaje de actividades que promuevan el desarrollo fonológico, la conciencia de letras o el aprendizaje de nuevo vocabulario:

6. Variables de identificación familiar:

Nombres y apellidos del padre y madre:

Edad: Estudios: Profesión:

Tipo de vivienda: Hermanos: Puesto que ocupa entre los hermanos:

Convive con algún familiar más:

\section{Discusión}

En relación a la literatura existente sobre las dificultades de aprendizaje en la lectoescritura, nos encontramos con la existencia de diversos modelos con metodologías y teorías diferentes (como por ejemplo el modelo del déficit o el modelo de respuesta a la intervención); diversos métodos de enseñanza de la lectura y la escritura, así como diversos autores con posturas contrarias. A su vez, algunos autores consideran la dislexia como una categoría, mientras otros, como Shankweiler y cols. (1996) o Prior (1989) (citado por Pogorzelski y Wheldall, 2005) la conceptualizan como una dimensión.

Se observa la necesidad de realizar estudios que incluyan diferentes subtipos de dislexia, así como diferentes grupos por edades, dado que, algunos autores como Thompson y cols. (2005) han observado que existen diferentes predictores a diferentes edades. Por ejemplo, el retraso en el lenguaje no aparece como un buen predictor hasta los cinco años y medio aproximadamente.

Igualmente, es fundamental la realización de estudios longitudinales, de estudios que analicen diferencias particulares entre lenguas opacas y lenguas transparentes, de estudios que se centren en variables socioafectivas; así como el establecimiento de un consenso entre los investigadores, tal y como defienden Aguilar et al. (2010), acerca de los procedimientos y recursos utilizados para analizar las variables implicadas en los procesos de lectura y sus predictores.
De acuerdo con Suárez, Manso y Godoy (2013), muchos estudios examinan las variables precursoras de la lectoescritura al mismo tiempo que miden la lectura, por lo que no puede valorarse la relación entre dichas variables. Por ello, se vuelven necesarios los estudios que investiguen las variables antes de que los niños reciban una enseñanza formal y sistemática en la lectura y la escritura.

Cuanto más conocimiento se tenga, mejores evaluaciones podrán hacerse, tratando de acortar así su duración, pues hoy en día suelen ser procesos largos y pesados, lo cual no motiva al sujeto.

Por último, destacar que en la práctica es indispensable el trabajo multidisciplinar y conjunto entre los distintos profesionales, así como con la familia. Además, el sistema escolar debe reconocer que existen diversos estilos de aprendizaje, cada uno único e irrepetible y debe promover un espacio donde se reconozcan tales diferencias.

\section{Referencias}

Aguilar Villagrán, M., Navarro Guzmán, J. I., Menacho Jiménez, I., Alcalde Cuevas, C., Marchena Consejero, E., y Ramiro Olivier, P. (2010). Velocidad de nombrar y conciencia fonológica en el aprendizaje inicial de la lectura. Psicothema, 22(3), 436-442.

American Psychiatric Association (APA). (2013). DSM-5 Diagnostic and statistical manual of mental disorders (5th ed.). Arlington, VA: American Psychiatric Publishing (Trad. Cast. (2014). DSM -5 Manual Diagnóstico y Estadístico de los Trastornos Mentales ( $5^{\mathrm{a}}$ Ed). Madrid: Editorial Médica Panamericana).

Báez Ávila, L., Rodríguez Arocho, W. C., y Martínez Mejías, S. (2009). Acercamiento a las Inhabilidades en el Aprendizaje desde una Neuropsicología Compleja. Informes Psicológicos, 11(12), 89-109.

Camargo, G. S. (2010). Estudio descriptivo de una familia disléxica y la herencia como factor. Revista Internacional de Investigación en Ciencias Sociales, 6(1), 7-36.

Carboni-Román, A., Del Río, D., Capilla, A., Maestú, F., y Ortiz, T. (2006). Bases neurobiológicas de las dificultades de aprendizaje. Revista de Neurología, 42(2), 171- 175.

D'Amato, R. C., Crepeau Hobson, F., Geil, M., D'Amato, R., y Huang, L. (2005). Ecological Neuropsychology: An alternative to the deficit model for conceptualizing and serving students with learning disabilities. Neuropsychology Review, 15(2), 97-103. doi:10.1007/s11065-005-7092-5

De Ramírez, R. A. (2000). Dificultades de aprendizaje de la lectura y la escritura. Educere Artículos, 4(11), 147-150.

Galaburda, A. M., y Cestnick, L. (2003). Dislexia del desarrollo. Revista de Neurología, 36(1), 3-9.

Gallagher, A., Frith, U., y Snowling, M. J. (2000). Precursors of Literacy Delay among Children at Genetic Risk of Dyslexia. Journal of Child Psychology and Psychiatry, 41(2), 203-213. doi:10.1111/1469-7610.00601 
Jiménez, J. E., Artiles, C., Rodríguez, C., Naranja, F., González, D., Crespo, P., Hernández, A., y Afonso, M. (2011). Dificultades específicas de aprendizaje: mirando hacia el futuro. Revista Electrónica de Dificultades de Aprendizaje, 1(1), 1-15.

Kavale, K. A., Holdnack, J. A., y Mostert, M. P. (2005). Responsiveness to intervention and the identification of specific learning disability: A critique and alternative proposal. Learning Disability Quarterly, 28(1), 2-16.

Manga, D., y Campos, F. R. (2011). El legado de luria y la neuropsicología escolar. Psychology, Society \& Education, 3(1), 1-13.

Pogorzelski, S., y Wheldall, K. (2005). The importance of phonological processing skills for older low-progress readers. Educational Psychology in Practice, 21(1), 1-22. doi:10.1080/02667360500035074103-111.

Preilowski, B., y Matute, E. (2011). Diagnóstico neuropsicológico y terapia del trastorno de lectura-escritura (dislexia del desarrollo). Revista Neuropsicología, Neuropsiquiatría y Neurociencias, 11(1), 95-122.

Restrepo, G. (2015). Transactional neuropsychology, a holistic view of children with developmental disabilities. Revista Ciencias De La Salud, 13(3), 431-445.

Suárez, A., Manso, J. M., y Godoy, M. J. (2010). Vocabulario y comprensión lectora: algo más que causa y efecto. Álabe, (1), 1-18. doi: http://dx.doi.org/10.15645/Alabe.2010.1.7

Suarez-Coalla, P., Garcia-de-Castro, M., y Cuetos, F. (2013). Variables predictoras de la lectura y la escritura en castellano. Infancia y Aprendizaje, 36(1), 77-89.

Thompson, P. A., Hulme, C., Nash, H. M., Gooch, D., Hayiou-Thomas, E., y Snowling, M. J. (2015). Developmental dyslexia: predicting individual risk. Journal of Child Psychology and Psychiatry 56(9), 976-987. doi: 10.1111/jcpp.12412 